\title{
RISK MANAGEMENT METHODS FOR COMMERCIAL CREDITS
}

\author{
Claudia ANTAL-VAIDA \\ The Bucharest University of Economic Studies, Romania \\ Claudia_antal@ymail.com
}

\begin{abstract}
Considering the current economic background, all economic entities are facing lots of challenges when setting up their growth strategies and they are often in the position to invest in new technologies to keep their competitive advantage. This paper reviews the impact of Digital Era in the Financial field and explains how these entities can leverage the advantages created by this trend, especially for credit risk management. Credit risk is one of the highest faced by banks and usually the one requiring the most capital, therefore the interest in predicting, measuring and minimizing it is of high interest for the banks. In order to understand the level of knowledge in the area, there were reviewed and summarized multiple articles written after the global crisis from 2007, outlining advantages and disadvantages of their applicability. However, it is difficult to say which performs best for classification purposes, because its success depends on multiple factors, including the accuracy, variety and complexity of the inputted dataset.
\end{abstract}

Keywords: Business Analytics, Consumer Credits, Credit Risk Assessment, Machine Learning, Scoring Models

JEL classification: $\mathrm{G} 24, \mathrm{O} 30$

DOI: $10.24818 / \mathrm{ie} 2020.04 .05$

\section{Introduction}

The current fast-paced and dynamic economic environment determines all the industries to adapt to change and adopt new technologies to remain competitive on the market. They are all facing various challenges and difficulties when establishing their growth strategy and in order to overcome them, they need to switch their focus when taking decisions, relying more on the data the company generates and not only on the human business judgement. If they want to embrace this new approach and become data-driven companies, they need to consider investing in data-processing power and methods of generating insights to get valuable information out of the historical facts. Change is everywhere and if we analyze the emerging growth of technologies, we will notice that data and data analysis are playing a key role in it. Hence, all business decision should be taken based on results generated by business analytics for a sustainable and ascending growth. Good news is that companies understood the impact and importance of this trend and acknowledged the business value it can bring when applied consistently. What's the definition of Business Analytics? Gartner defines it as a collection of practices focused on simulations and models that analyze the past facts to understand reality and gain insights to drive business planning, but also predict the go-forward state of the system. [1] At a glance, it can be described as an emergent business enabler that analyzes historical data and uses it to predict future trends and events. In the past years it has become a highly required area mainly due to its impact on the financial growth and profitability, leading to a better competitive advantage and to a reduced decision error. Its applicability was proven to be very effective in creating new opportunities, choosing a good timing and improving the business knowledge. The banking sector does not make an exception, therefore embracing the change and adopting business analytics is a must. These new techniques can be applied to better 
understand customer's behavior, improve interactions with him, understand his motivation to pay his debts and carry through their financial obligations, but also reduce the exposure to risks. The banking area seems very complex, but its main purpose and utility is to handle cash and credits and secure funds to produce more money. Considering that it mainly manages money, this industry is highly exposed to multiple risks, the major ones being operational risks, market risks and credit risks. Operational risk can happen due to a potential operational breakdown [2], that can be triggered by internal factors (deficient processes, people, system errors, human mistakes) or external one (economic crisis, fraud, global trends and unexpected events), the lastly mentioned being very hard to predict or identify. Market Risk appears for every player that invests, trades and plays on the financial markets. It is hard to eliminate, since in most of the cases is triggered by a global external event, but there are ways of diminishing it. Last but not least, one of the highest risks to which banks are exposed is Credit Risk which requires the most capital, hence the high interest in predicting, measuring and preventing it. The main objective of credit risk management is to optimize the credit portfolio and reduce the number of customers that have potential of not meeting their obligations in the future. All these types of risks are complex and have a significant impact on the financial institutions' growth. Machine learning and artificial intelligence's applicability increased consistently and started to play a key role in predicting these events, assessing their impact and minimizing their effects [3]. Banks inquire the assessment of the best ways to protect their data, systems and clients and machine learning has the potential to support that. Process automation can minimize human error, increase the execution of recurrent tasks, inspect data to outline the relevant insights and increases the ability to evaluate risky clients and networks. It can also trigger and prioritize alerts for unusual activities and asses the risk involved.

\section{Digital Transformation in the Banking area}

The following part of this paper explains the concept of big data and how companies decided to leverage the advantages created to improve their competitivity and financial results. The high amounts of data created each second increased the applicability of Business Analytics in business applications, with lots of solutions already implemented and many others being tested. The Digital Era is characterized by new technologies which increase the speed of knowledge turnover [4]. The growing influence of big data, analytics, cloud and social media caused overall disruption across industries, the Banking area being highly impacted. [5]

This new trend led to so much data, that human beings lost their ability to manually analyze it, reason why people started to look for alternatives and it facilitated the apparition of Data Mining, Big Data and Business Analytics. All these methods enable the ability to efficiently analyze data, easily identify patterns and convert data into information and insights that support and improve the business processes.

Business Analytics helps the upper management to easily understand the business, solve issues and make fact-based decisions [6], but also identify opportunities to address unexplored markets and extend activity. More than that, Business Analytics can help banks understand customers' behavior and their capacity of paying their debts, identify new products that would be more suited for customers' needs and reduce the impact of various risks. Figure 1 presents the four stages of Business Analytics and the correlation between effort and results: it is easy to notice that the business value brought is proportional with the complexity of the analysis performed. [7] 
www.conferenceie.ase.ro

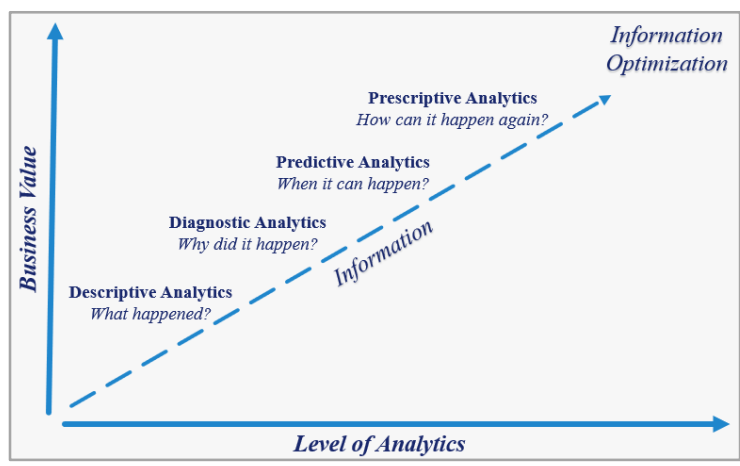

Figure 1. Stages of Business Analytics

Source: Understanding the Role of Business Analytics [7]

\section{Algorithms for Credit Risk Management}

Since the global financial crisis took place, Risk management has become more important in the banking fields and the focus on risks detection, measurement and management increased. [8] Back in the '90s, there was a study on traditional statistical models for bankruptcy and an alternative neural network algorithm revealed efficient results, with outstanding accuracy. [9] Over years, there were multiple researches on machine learning techniques for risk management, which outlined increased efficiency and better precision in recommending an optimal decision. For credit risk management, the most common tool used is the credit scoring model, that uses statistical methods to review financial indicators in order to predict the default risk of entities and their creditworthiness, pointing out the probability of default of the borrower. [8] The following part of this paper mainly focuses on the applications that were developed and deployed for consumer credits in the risk management area after the financial crisis, the most popular ones being the classification methods, which group the members of the dataset in clusters, based on their features. Table 1 contains a list of different classification techniques.

Table 1. Machine Learning algorithms in Credit Scoring

\begin{tabular}{|l|l|}
\hline Algorithm & Reference \\
\hline Artificial Neural Networks (ANNs) & {$[10][11][12]$} \\
\hline Bagging & {$[12]$} \\
\hline Bayesian classifier & {$[10][11]$} \\
\hline Boosting & {$[12]$} \\
\hline Decision Tree & {$[10][11][13]$} \\
\hline Deep Learning & {$[14]$} \\
\hline Discriminant Analysis & {$[10][11]$} \\
\hline Extreme Machine Learning & {$[15]$} \\
\hline Fuzzy rule-based system & {$[10]$} \\
\hline Hybrid Models & {$[10]$} \\
\hline K-Nearest Neighbor (K-NN) & {$[10][11]$} \\
\hline Lasso logic regression & {$[16]$} \\
\hline Logistic regression & {$[10][11][15]$} \\
\hline Random forest & {$[11][12][17]$} \\
\hline Support Vector Machines (SVM) & {$[10][11][15][18][19][20]$} \\
\hline Survival Analysis & {$[10]$} \\
\hline
\end{tabular}


After reading all these articles on machine learning algorithms, the following observations were withdrawn:

- Artificial Neural Networks (ANNs) are non-linear statistical models based on human-brain function, very efficient for unknown data relationship modeling. They have the ability to identify complex patterns and predict the result of new independent data.

- Bagging is an algorithm that uses bootstrapping to generate a training set from the initial input. The classification results are combined and arranged to improve the accuracy of the prediction. An advantage of this method is that learning can be performed independently, due to the mutual independence of the bootstrap.

- Bayesian classifier, mainly suited when the input's dimensionality is high, is a simple probabilistic classifier based on Bayes' theorem. It assumes that the existence or nonexistence of a characteristic of a group is not corelated with the existence or nonexistence of any other feature.

- Boosting is a technique that implies sequential learning and generates multiple results that are finally integrated to improve overall accuracy.

- Decision Tree uses tree-like graphs, covering all possible scenarios that derive out of all possibilities, the top-node being the input and the leaf nodes being the final possible groups. One of its major advantages is that the output is easy to understand and verify, but a big disadvantage is the inability of scaling it to different contexts.

- Deep Learning uses multiple processing layers with complex structure or composed of nonlinear transformations. The model has a basic unit, a neuron, inspired by human brains which is activated by a function. These models can learn useful representations of unprocessed data and have high performance on complex data like videos, images and text.

- Discriminant Analysis, an alternative to the logistic regression, starts from the premises that the explanatory variables have a multivariate normal distribution. It mainly focuses on reducing the distance within member of the groups and increase the distance between the groups themselves.

- Extreme Machine Learning is a hidden layer feed-forward network that is known for its efficiency, providing accurate results in a short training timeframe.

- Fuzzy rule-based system focuses on designing rules to derive the credit score with details, despite other models that focus on estimating the scores. Its major advantage is the ability to handle both quantitative and qualitative features, so the results will be less sensitive to measurement errors.

- Hybrid Models represent mixes of two or more algorithms and their main advantage is that they bring in the positive aspects from multiple algorithms and can remove disadvantages of individual models.

- K-Nearest Neighbor (K-NN) is a nonparametric classifier that learns from similarities. It uses a distance function for the explanatory features and once a new observation is fed into the model, it reviews its pattern looking into the $\mathrm{K}$ nearest neighbors and is assigned to the class which is closer.

- Lasso logic regression estimates the coefficient of the regression by maximizing the loglikelihood function in order to improve the prediction accuracy and interpretability of the result.

- Logistic regression is a form of linear regression that do not assume linearity of relationship between independent variables and the dependent ones do not require a normal distribution of variables. It can be applied on mix of variables, no matter if they are discrete, continuous or binary. 
- Random forest is a classifying and regression method that uses multiple decision trees for training and results in a class that represents the mode of the classes or the mean prediction of the trees. The author used weights for decision trees, calculated based on the previous performance, namely errors in training. The major advantage outlined was the reduced time for training, mainly because of the parallel run.

- Support Vector Machines (SVM) is a technique that involve 3 elements: a linear combination of selected characteristics, an objective function that evaluates both training and test data and an algorithm for determining the optimal parameters. Its main particularity is that it focuses more on extreme cases that are more similar with the opposite boundary. This algorithm proved to be very efficient in the classification area and when they are combined with genetic algorithms, they can successfully serve multiple purposes: performing feature selection tasks but also optimize the model parameters. Another study revealed that they can be well applied selecting the features that have the highest impact on likelihood of default.

- Survival Analysis can compute the profitability of a customer over its lifetime and calculate profit scoring. It mainly focuses on predicting the time until an event will happen, more than its probability of occurrence.

It is easy to notice that the most researched algorithms were SVMs, Random Forest, Decision Trees, Logistic Regression and Artificial Neural Networks, that have also become benchmarks in the classification area. However, it is hard to assess which one provides the best classification results considering that the analysis was performed on different datasets, hence the performance was dependent on multiple factors, not only on the algorithm itself.

\section{Conclusions}

The world is changing and companies cannot rely anymore on traditional practices to run the business. If an entity wants to remain competitive and survive on the market, the only option is to stay in line with new trends, adopt new practices and adapt the activity to the fast-paced economic background. The same applies to financial institutions: they need to adapt, but also find ways to identify all the risks to each they are exposed in order to minimize financial loss. The articles reviewed are detailing the credit risk management thru different techniques, mainly outlining the attention gained by this area in the past years. Even though the observations and conclusions obtained by the authors can be further leveraged in analysis, it is difficult to identify only one algorithm that can be recommended for this purpose. The only obvious output would be that all presented algorithms outperformed the traditional ones, not only in terms of accuracy, but also by optimizing the running time and resources consumed.

Another important point is that even if the advantages are multiple and the business value add is significantly higher than traditional techniques, the limitations and disadvantages of Machine Learning should be acknowledged when deciding to adopt it. Not to forget that the accuracy of the model is highly dependent on the external variables that cannot be predicted all the times and also on the dataset used for training: it should be varied, integer, divers, to cover as many scenarios as possible and reduce biased results.

In conclusion, even though there have been different studies performed in this area, there's still room for improvement to extend the beneficial applicability and impact of machine learning in the financial field.

\section{References}

[1] "Gartner Glossary", Gartner, Internet: https://www.gartner.com/en/informationtechnology/glossary/business-analytics

[2] I. A. Moosa, Operational Risk Management, Palgrave MACMILLAN, 2007. 
[3] T.-M. Choi, H. K. Chan and X. Yue, "Recent Development in Big Data Analytics for Business Operations and Risk Management," IEEE Transactions on Cybernetics, 2007.

[4] G. Doukidis, N. Mylonopoulos and N. Pouloudi, Social and Economic Transformation in the Digital Era, IGI Global, 2004.

[5] B. Raghynathan and R. V. Maiya, SMACing the Bank - How to Use Social Media, Mobility, Analytics, and Cloud Technologies to Transform the Business Processes of Banks and the banking Experience, CRC Press, 2018.

[6] J. R. Evans, Business Analytics - Methods, Models and Decisions, Second Edition, Pearson Education, Inc., 2016.

[7] H. Chahal, J. Jyoti and J. Wirtz, Understanding the Role of Business Analytics, Spinger, 2019.

[8] L. Martin, S. Suneel and K. Maddulety, "Machine Learning in Banking Risk Management: A Literature Review," Risks, 2019.

[9] E. I. Altman, M. Giancarlo and F. Varetto, "Corporate distress diagnosis: Comparison using linear discriminant analysis and neural networks (the Italian experience)," Journal of Banking \& Finance, 1994.

[10] A. Keramati and N. Yousefi, "A Proposed Classification of Data Mining Techniques in Credit Scoring," in International Conference on Industrial Engineering and Operations Management, Kuala Lumbur, Malaysia, 2011.

[11] S. Lessman, B. Baesens, H.-V. Seow and L. C. Thomas, "Benchmarking state-of-the-art classipcation algorithms for credit scoring: An update of research," European Journal of Operational Research, 2015.

[12] S. Hamori, M. Kawai, T. Kume, Y. Murakami and C. Watanabe, "Ensemble Learning or Deep Learning? Application to Default Risk Analysis," Journal of Risk and Financial Management, 2019.

[13] A. E. Khandani, K. J. Adlar and L. W. Andrew, "Consumer credit-risk models via Machine Learning Algorithms," Journal of banking \& finance , 2010.

[14] V.-S. Ha and H.-N. Nguyen, "Credit scoring with a feature selection approach based deep learning," MATEC Web of Conference, vol. 54, 2016.

[15] L. Yu, Z. Yang and L. Tang, "A Novel Multistage Deep Belief Network Based Extreme Learning Machine Ensemble Learning Paradigms for Credit Risk Assessment," Flexible Services and Manufacturing Journal, vol. 28, 2016.

[16] H. Wang, Q. Xu and L. Zhou, "Large Unbalanced Credit Scoring Using Lasso-Logistic Regression Ensemble," PloS Ones, 2015.

[17] L. Zhou and H. Wang, "Loan Default Prediction on Large Imbalanced Data Using Random Forests," TELKOMNIKA Indonesian Journal of Electrical Engineering, vol. 10, pp. 1519$1525,2012$.

[18] C. L. Huang, M. C. Chen and C. J. Wang, "Credit scoring with a data mining approach beased on support vector machines," ScienceDirect - Expert Systems with Applications, vol. 33, pp. 847-856, 2007.

[19] T. Bellotti and J. Crook, "Support vector machines for credit scoring and discovery of significant features," ScienceDirect - Expert Systems with Applications, vol. 36, pp. 33023308, 2009.

[20] T. Harris, "Quantitative credit risk assessment using support vector nachines: Broad versus Narrow deafult definitions," Elsevier - Expert Systems with Applications, vol. 40, 2013. 